\title{
Entendiendo los monocitos para rastrear la tuberculosis
}

\author{
Understanding monocytes to trace tuberculosis
}

D

ebido al gran número de casos nuevos de tuberculosis, la emergencia de cepas resistente y las comorbilidades que comprometen la función del sistema inmune [1], esta condición patológica tiene cada vez mayor impacto en el ámbito global. A pesar de las múltiples investigaciones, la complejidad del problema y los impactos sociales y económicos hacen de la tuberculosis una enfermedad que se considera devastadora. Según la Organización Mundial de la Salud (OMS) se estima que una cuarta parte de la población mundial tiene tuberculosis latente; término aplicado para las personas infectadas por $\mathrm{My}$ cobacterium tuberculosis que aún no han enfermado ni pueden transmitir la infección. La tuberculosis, durante muchos años, y también según las estadísticas de la OMS, está dentro de las diez primeras causas de muerte en el mundo [2]. Si se contrasta con otras enfermedades infecciosas, también devastadoras, como la diarrea y las enfermedades respiratorias, que pueden ser originadas por múltiples agentes etiológicos, la tuberculosis es la enfermedad más importante causada por un único agente etiológico.

La infección usualmente comienza por el tracto respiratorio, donde las bacterias inhaladas pueden ser fagocitadas por los macrófagos residentes del alvéolo, los cuales son los responsables de activar los mecanismos tempranos de la respuesta innata [3]. Esta respuesta compromete otros tipos de células como las dendríticas y los monocitos, reclutados de la corriente sanguínea, e incluso subpoblaciones de NK $[4,5]$ y neutrófilos [6,7]. Las interacciones iniciales entre la micobacteria y las células hospederas fagocíticas han sido consideradas como eventos capitales en las fases ulteriores de la respuesta inmune $[8,9]$. Por ello, dadas las restricciones para acceder de manera expedita a muestras de pulmón, estas interacciones han sido estudiadas, por ejemplo, en nuestro caso, utilizando fagocitos obtenidos principalmente de sangre periférica, promonocitos humanos [10-13] y macrófagos esplénicos tanto humanos[14,15] como múridos [16].

Nuestras observaciones han hecho evidentes varias diferencias entre los fagocitos mononucleares de los pacientes con tuberculosis y los individuos sanos. Una de las más notorias consiste en que los monocitos de los pacientes, cuando son infectados in vitro con Mycobacterium tuberculosis H37Rv o tratados con derivado proteico purificado (PPD), una fracción de ellos muere con evidentes alteraciones en la membrana celular y otra con daño mitocondrial, exposición de la fosfatidilserina, activación de caspasas y daño en el ADN. En el caso de los monocitos de los controles sanos se observa fundamentalmente muerte con daño mitocondrial y una escasa proporción de células con daño en la membrana [11,12].

Estas evidencias llevaron a nuestro grupo de Investigación a evaluar si los mecanismos efectores de los fagocitos mononucleares estaban asociados con los de la muerte celular, 
cuyos hallazgos indicaron una asociación entre dos citoquinas: el TNF- $\alpha$, que modula la muerte con daño mitocondrial y actividad de caspasas [12,17], y la IL-10, que se asocia con los mecanismos de muerte con daño en la membrana celular.

Además, hallamos que, mientras la inhibición de las vías efectoras de la apoptosis no tuvo ninguna repercusión en la supervivencia de la micobacteria, el bloqueo de las vías con daño en la membrana mostró que esta era dependiente de la actividad de una forma soluble de la fosfolipasa A-2 (PLA-2) y que su bloqueo favorecía el control de la bacteria. De notarse, el principal sustrato de la actividad de esta enzima, el ácido araquidónico, es capaz de inducir muerte con daño en la membrana celular en los fagocitos $[18,19]$.

La participación de la PLA-2 en los mecanismos de muerte, así como la activación diferencial de vías de muerte entre células de pacientes y controles, nos llevó a considerar que los fagocitos, probablemente, también podrían tener diferencias en su composición lipídica. En esta ocasión encontramos que los ácidos grasos de las membranas de las células más susceptibles a la muerte por daño en la membrana son en mayor proporción insaturados y diferenciales entre las subpoblaciones de monocitos [20].

Además, se encontró que la composición de ácidos grasos totales de los fagocitos también cambia con la diferenciación y la infección [20] y, más recientemente, usando un sistema in vitro que solo incluye a los fagocitos mononucleares, que estos tienen mayor acumulación de ácido esteárico cuando se han diferenciado en presencia de Mycobacterium tuberculosis a bajas cantidades de bacterias por cada célula. Estas observaciones llevaron a considerar que el estudio de los ácidos grasos provenientes de las vías aéreas inferiores [21], que lograría dar cuenta del ambiente en los sacos alveolares y bronquios, podría tener algún vestigio que ayude a definir a los individuos infectados o que directamente marque la presencia de Mycobacterium tuberculosis.

Esta definición del ambiente inflamatorio de las vías inferiores emerge de la necesidad de detectar más temprana y eficientemente la bacteria, a fin de poder iniciar el tratamiento lo antes posible. Clásicamente, el diagnóstico de la tuberculosis se hace mediante la detección de los bacilos con la tinción de Ziehl-Neelsen de forma directa o mediante el prolongado cultivo. No obstante, en algunos individuos infectados, con una respuesta inmune no "típica", los bacilos no son evidentes. Estos individuos incluyen a los denominados paucibacilares, entre los cuales se encuentran los niños [22]. La detección tardía de la bacteria prolonga el inicio del tratamiento definitivo y certero, lo que lleva al deterioro de la salud de los pacientes y aumenta el riesgo de trasmisión.

Derivado de la dificultad de detectar la micobacteria sobresale la dificultad para diagnosticar a los adultos paucibacilares y niños con tuberculosis pulmonar. Por tal razón, la OMS, dentro de la estrategia "Alto a la Tuberculosis" [23], sugiere la realización de investigaciones para mejorar y promover nuevas herramientas que permitan realizar un diagnóstico rápido y confiable, y así efectuar el tratamiento oportuno que mejore la salud del paciente y disminuya la propagación de la infección.

Con base en los diferentes estudios que avalan el uso del condensado de aire exhalado 
(EBC; del inglés, exhaled breath condensate) como una fuente válida de las vías aéreas inferiores $[21,24,25]$ y en las evidencias in vitro que han permitido discriminar el comportamiento de los fagocitos mononucleares provenientes de pacientes con tuberculosis de los de individuos sanos, mediante el análisis del perfil de ácidos grasos, citoquinas y quimioquinas [20], sumado a la secreción activa de glicolípidos hacia el espacio extracelular por parte de Mycobacterium tuberculosis [26,27], se propuso estudiar el ambiente pulmonar de los pacientes adultos bacilíferos y paucibacilares, y de niños con tuberculosis pulmonar, a través de los niveles de ácidos grasos, citoquinas y quimioquinas presentes en los condensados de aire exhalado [21].

Para el desarrollo de dicha investigación fue necesario adentrarnos en la literatura, a fin de comprender los métodos que actualmente se han propuesto y tratado de desarrollar para el diagnóstico de la tuberculosis, incluyendo el análisis de los componentes y derivados lípidicos de la micobacteria; revisión que se entrega a la comunidad médica y personal de la salud en el presente número de Medicina \& LABORATORIO.

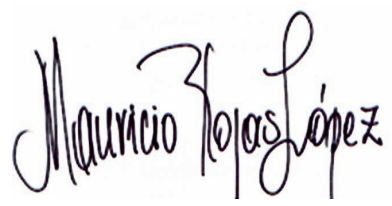

\section{Mauricio Rojas López. Biol, MSc, PhD.}

Profesor e investigador, Grupo de Inmunología Celular e Inmunogenética, Universidad de Antioquia.

Coordinador Unidad de Citometría de Flujo, Sede de Investigación Universitaria, Universidad de Antioquia.

Medellín, Colombia, diciembre de 2017.

\section{Bibliografía}

1. World Health Organization. Global tuberculosis report 2017. Ginebra, Suiza: World Health Organization; 2017.

2. World Health Organization. The top 10 causes of death. 2017. Disponible: http://www.who.int/en/news-room/ fact-sheets/detail/the-top-10-causes-of-death. Consultado: dic 2017.

3. Magee DA, Conlon KM, Nalpas NC, Browne JA, Pirson C, Healy C, et al. Innate cytokine profiling of bovine alveolar macrophages reveals commonalities and divergence in the response to Mycobacterium bovis and Mycobacterium tuberculosis infection. Tuberculosis (Edinb) 2014; 94: 441-450.

4. Lande R, Giacomini E, Grassi T, Remoli ME, Iona E, Miettinen M, et al. IFN-alpha beta released by Mycobacterium tuberculosis-infected human dendritic cells induces the expression of CXCL10: selective recruitment of NK and activated T cells. J Immunol 2003; 170: 1174-1182.

5. Perskvist $\mathbf{N}$, Long $\mathbf{M}$, Stendahl $\mathrm{O}$, Zheng L. Mycobacterium tuberculosis promotes apoptosis in human neutrophils by activating caspase-3 and altering expression of Bax/Bcl-xL via an oxygen-dependent pathway. J Immunol 2002; 168: 6358-6365. 
6. Pedrosa J, Saunders BM, Appelberg R, Orme IM, Silva MT, Cooper AM. Neutrophils play a protective nonphagocytic role in systemic Mycobacterium tuberculosis infection of mice. Infect Immun 2000; 68: 577-583.

7. Gonzalez-Cano P, Mondragon-Flores R, Sanchez-Torres LE, Gonzalez-Pozos S, Silva-Miranda M, Monroy-Ostria A, et al. Mycobacterium tuberculosis H37Rv induces ectosome release in human polymorphonuclear neutrophils. Tuberculosis (Edinb) 2010; 90: 125-134.

8. Salam N, Gupta S, Sharma S, Pahujani S, Sinha A, Saxena RK, et al. Protective immunity to Mycobacterium tuberculosis infection by chemokine and cytokine conditioned CFP-10 differentiated dendritic cells. PLoS One 2008; 3 : e2869.

9. Tascon RE, Soares CS, Ragno S, Stavropoulos E, Hirst EM, Colston MJ. Mycobacterium tuberculosis-activated dendritic cells induce protective immunity in mice. Immunology 2000; 99: 473-480.

10. Castano D, Barrera LF, Rojas M. Mycobacterium tuberculosis alters the differentiation of monocytes into macrophages in vitro. Cell Immunol 2011; 268: 60-67.

11. Castano D, Garcia LF, Rojas M. Increased frequency and cell death of CD16+ monocytes with Mycobacterium tuberculosis infection. Tuberculosis (Edinb) 2011; 91: 348-360.

12. Gil DP, Leon LG, Correa LI, Maya JR, Paris SC, Garcia LF, et al. Differential induction of apoptosis and necrosis in monocytes from patients with tuberculosis and healthy control subjects. J Infect Dis 2004; 189: 2120-2128.

13. Mosquera-Restrepo SF, Caro AC, Pelaez-Jaramillo CA, Rojas M. Mononuclear phagocyte accumulates a stearic acid derivative during differentiation into macrophages. Effects of stearic acid on macrophage differentiation and Mycobacterium tuberculosis control. Cell Immunol 2016; 303: 24-33.

14. Duque C, Arroyo L, Ortega H, Montufar F, Ortiz B, Rojas M, et al. Different responses of human mononuclear phagocyte populations to Mycobacterium tuberculosis. Tuberculosis (Edinb) 2014; 94: 111-122.

15. Henao J, Sanchez D, Munoz CH, Mejia N, Arias MA, Garcia LF, et al. Human splenic macrophages as a model for in vitro infection with Mycobacterium tuberculosis. Tuberculosis (Edinb) 2007; 87: 509-517.

16. Rojas M, Barrera LF, Puzo G, Garcia LF. Differential induction of apoptosis by virulent Mycobacterium tuberculosis in resistant and susceptible murine macrophages: role of nitric oxide and mycobacterial products. J Immunol 1997; 159: 1352-1361.

17. Rojas M, Olivier M, Gros P, Barrera LF, Garcia LF. TNF-alpha and IL-10 modulate the induction of apoptosis by virulent Mycobacterium tuberculosis in murine macrophages. J Immunol 1999; 162: 6122-6131.

18. Arcila ML, Sanchez MD, Ortiz B, Barrera LF, Garcia LF, Rojas M. Activation of apoptosis, but not necrosis, during Mycobacterium tuberculosis infection correlated with decreased bacterial growth: role of TNF-alpha, IL-10, caspases and phospholipase A2. Cell Immunol 2007; 249: 80-93.

19. Escobar-Alvarez E, Pelaez CA, Garcia LF, Rojas M. Human monocyte differentiation stage affects response to arachidonic acid. Cell Immunol 2010; 264: 61-70.

20. Ramirez-Agudelo ME, Caro AC, Jaramillo CA, Rojas M. Fatty acid profile during the differentiation and infection with Mycobacterium tuberculosis of mononuclear phagocytes of patients with TB and healthy individuals. Cell Immunol 2011; 270: 145-155.

21. Mosquera-Restrepo SF, Caro AC, Garcia LF, Pelaez-Jaramillo CA, Rojas M. Fatty acid derivative, chemokine, and cytokine profiles in exhaled breath condensates can differentiate adult and children paucibacillary tuberculosis patients. J Breath Res 2017; 11: 016003.

22. Westaway MS, Wessie GM. Tuberculosis diagnosis and treatment of young South African children: experiences and perceptions of care-givers. Tuber Lung Dis 1994; 75: 70-74.

23. World Health Organization. WHO launches new stop TB strategy to fight the global tuberculosis epidemic. Indian J Med Sci 2006; 60: 125-126.

24. Khoubnasabjafari M, Ansarin K, Jouyban-Gharamaleki V, Panahi-Azar V, Hamidi S, Azarmir Z, et al. Methadone Concentrations in Exhaled Breath Condensate, Serum and Urine of Patients Under Maintenance Treatment. Iran J Pharm Res 2017; 16: 1621-1630.

25. Kwiatkowska S, Szkudlarek U, Luczynska M, Nowak D, Zieba M. Elevated exhalation of hydrogen peroxide and circulating IL-18 in patients with pulmonary tuberculosis. Respir Med 2007; 101: 574-580.

26. Bhatnagar S, Schorey JS. Exosomes released from infected macrophages contain Mycobacterium avium glycopeptidolipids and are proinflammatory. J Biol Chem 2007; 282: 25779-25789.

27. Bhatnagar S, Shinagawa K, Castellino FJ, Schorey JS. Exosomes released from macrophages infected with intracellular pathogens stimulate a proinflammatory response in vitro and in vivo. Blood 2007; 110: 3234-3244. 